\title{
DEVELOPMENT OF CONSTITUTIVE MODELS FOR FAST REACTOR DESIGN
}

\author{
K. Tsukimori ${ }^{1}$, K. Iwata $^{2}$, N. Kawasaki ${ }^{1}$, N. Kasahara ${ }^{3}$ \\ ${ }^{1}$ Advanced Nuclear System Research and Development Directorate, Japan Atomic Energy Agency, Ibaraki, JAPAN \\ ${ }^{2}$ Visiting Researcher, Japan Atomic Energy Agency, Ibaraki, JAPAN \\ ${ }^{3}$ Invited Researcher, Japan Atomic Energy Agency/ University of Tokyo, Tokyo, JAPAN \\ E-mail of corresponding author: tsukimori.kazuyuki@jaea.go.jp
}

\begin{abstract}
$\mathrm{R} \& \mathrm{D}$ to enable a practical fast breeder reactor plant is proceeding in Japan, which is called "FaCT (Fast reactor Cycle Technology development). One of the key issues of $R \& D$ is to realize a reasonably small reactor vessel by eliminating the thermal liner which is installed inside the vessel in order to reduce thermal loading in the conventional design. Most important concern is the amount of the inelastic strain of the vessel accumulated around the liquid sodium surface which moves up and downward cyclically with start-up and shut-down. The aim of this study is to develop rational constitutive models that enable prediction of this kind of complex inelastic behaviors precisely and to prepare the design guide based on inelastic analysis. In this paper, the R\&D results are introduced.
\end{abstract}

\section{INTRODUCTION}

R\&D to enable a practical fast breeder reactor plant is proceeding in Japan. One of the key issues of R\&D is to realize a reasonably small reactor vessel by eliminating the thermal liner which is installed inside the vessel in order to reduce thermal loading in the conventional design. Most important concern is the amount of the inelastic strain of the vessel accumulated around the liquid sodium surface which moves up and downward cyclically with start-up and shut-down. The aim of this study is to develop rational constitutive models that enable prediction of this kind of complex inelastic behaviors precisely and to prepare the design guide based on inelastic analysis[1].

The requirements for the target constitutive models are as follows.

(1) expression of the nonlinearity of stress-strain relation of the material (316FR in this study)

(2) applicability to cyclic loading conditions in the elastic plastic region

(3) prediction of stress- strain behavior properly under the temperature changing conditions ( $~ 600$ deg. C )

(4) applicability to non-proportional multi-axial behaviors

Furthermore, it must be promised that the design evaluations give conservative results.

In order to develop the constitutive models that satisfy these requirements, we proceeded a project consisting of the following five parts.

(i) Simulation analyses of the inelastic stress-strain behavior of the reactor vessel made of 316FR by using the existing analysis methods ; The aim is to understand the mechanism of the strain accumulation in the liquid surface traveling region including the effect of the primary stress and grasp the characteristic stress-straintemperature behaviors and their ranges to be covered by the constitutive models roughly.

(ii) Development of candidate constitutive models ; The formulation of some constitutive models was implemented and basic check was done partially by comparing with the existing studies and the verification tests. We are preparing two kinds of models considering practical use, one is a simple and convenient model and the other is a detailed and precise model. Concerning the expression of the nonlinearity of stress-strain relation, two methods are pursued, i.e., the multi-linear approximation based on the multilayer kinematic hardening model[2] and the smooth curve approximation based on the two-surface model[3].

(iii) Incorporation of candidate constitutive models into the general purpose structural analysis system ; Since large scale analyses are needed in the practical design, the incorporation of the constitutive models and the study of effective numerical procedures are proceeded simultaneously.

(iv) Material property tests : These tests were conducted to get the basic property data of the target material and to determine the constants of constitutive models.

(v) Verification tests for the validity of the constitutive models ; Uniaxial tests with strain and temperature controlled under monotonic / cyclic loadings, biaxial tests with non-proportional loadings and structural element tests are conducted. The uniaxial and the biaxial tests were composed by introducing the characteristic behaviors obtained from the above simulation analyses of the reactor vessel. The structural element tests aim at verification of the constitutive models, focusing on the ratcheting phenomena. 
This R\&D started from late 2006FY and finished at the end of 2009FY. Fig. 1 shows the main items and the master program of the project.

\begin{tabular}{|c|c|c|c|c|}
\hline & 2006FY & 2007FY & $2008 F Y$ & 2009FY \\
\hline Simulation Analysis of RV & \multicolumn{4}{|c|}{ Preliminary Analysis $\quad$ Application Analysis } \\
\hline Development of Constitutive Models & \multicolumn{4}{|c|}{ Simple Model / Precise Constitutive Model } \\
\hline $\begin{array}{l}\text { Incorporation of Constitutive Models } \\
\text { into FEM code }\end{array}$ & \multicolumn{2}{|c|}{ Incorporation of models } & ation Analy & \\
\hline Material Property Tests & \multicolumn{3}{|c|}{ Basic material Data } & \\
\hline Verification Tests & \multicolumn{4}{|c|}{ Uni-axial test/ 3 bar model / Bi-axial model/ Cylindrical model } \\
\hline
\end{tabular}

Fig. 1: Main items and master program of the project

\section{PRELIMINARY ANALYSIS OF RATCHETING IN REACTOR VESSEL}

Fig. 2 shows the reactor vessel structure of the current demonstration FBR plant design in Japan and the thermal transient behaviors at start-up and shut down. At start-up, the temperature of sodium goes up from $200 \mathrm{C}$ to $550 \mathrm{C}$ and liquid surface goes up more than $1000 \mathrm{~mm}$, simultaneously. At shut down, the temperature distribution change is more complicated. The liquid temperature starts to descend from lower position, but the liquid near the surface cannot follow quickly. Consequently, during the thermal cycle, the maximum stress of the vessel is generated near the liquid surface (NSL-50mm). In the design this event is supposed to occur several hundred times during the life, and the ratcheting phenomenon near the liquid surface is concerned. It is one of the key issues of R\&D how to control the excessive strains accumulated due to this phenomenon. The details of the preliminary analysis are described in the previous paper [1].

The response of structure obtained by the analysis is complicated. Circumferential and axial components of stress and strain are dominant but the ratio of the two components is not constant at the center of thickness near the liquid surface. In other words, the relation between circumferential and axial stresses is non-proportional. And parametric analyses proved that the relation between primary and secondary stresses was affected by the thickness of the vessel, the internal pressure and the weight. The axial strain increases with cycles, while the circumferential strain decreases. And this behavior is accelerated by largeness of thickness and weight, while it is decelerated by internal pressure. The requirements for the constitutive models in scope and the following verification tests were constructed considering the preliminary analysis results

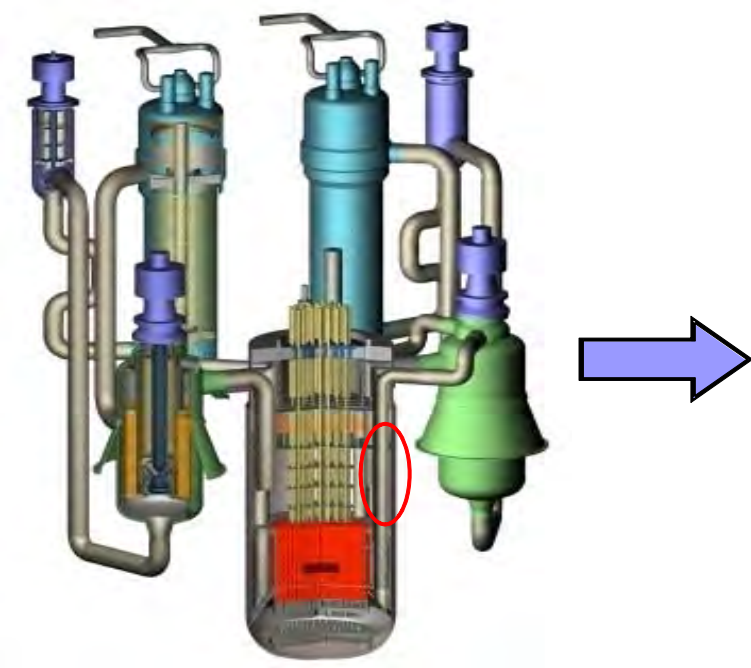

\section{Thermal loads}

Main loads are thermal stresses induced by fluid temperature change at transient operation

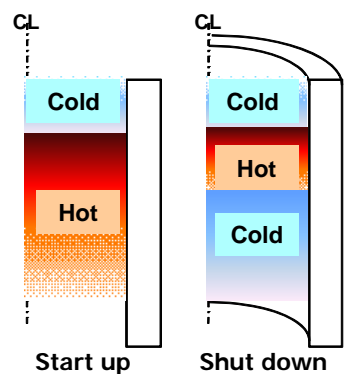

Fig. 2: Reactor Vessel structure of current demonstration FBR design and thermal transient behaviours 


\section{DEVELOPMENT OF ADVACED CONSTITUTIVE EQUATIONS FOR DESIGN}

\section{Simplified Plasticity Model (MK-SRR model)}

As a simplified plasticity model, the multi-linear kinematic hardening model with stress reversal-on resetting (MK-SRR model) was developed by incorporating a hardening reset procedure into the multi-linear kinematic hardening model (MK model) at every stress reversal event [1]. This model is recommended as a definitely conservative approach [4]. The concept of the model is that the hardening of a material recovers whenever a definite stress reversal occurs in cyclic loading and after the complete recovery the material restarts hardening following the initial monotonic stress-strain relation. MK-SRR model employing multi-linear stress-strain relations can represent stress-strain behavior more precisely than the bilinear kinematic hardening model.

\section{High-Accuracy Plasticity Model}

We developed two types of temperature dependent cyclic plasticity models. The two-surface cyclic plasticity model proposed by Iwata [3] and the multi-linear kinematic hardening model proposed by Ohno and Wang [2] were judged to be most promising to extend to allow for the analysis considering cyclic hardening as well as temperature dependency of material properties. The essence of the developed models is briefly outlined below.

\section{Modeling of cyclic hardening}

In addition to monotonic stress-plastic strain properties, cyclic steady state stress-plastic strain properties which are obtained from constant stress range cyclic tests are used as fundamental properties of cyclic plasticity. The original two-surface cyclic plasticity model [3] represented cyclic hardening by a simple evolution equation of plastic hardening modulus. An extended modeling approach for cyclic hardening based on arbitrary stress-strain relations was proposed to create cyclic plasticity models [5]. To describe cyclic stress-plastic strain relations, an equation called generic stress-plastic strain relation (see Fig. 3) was introduced as

$$
\begin{aligned}
& \sigma=\sigma_{Y}(T)+\varphi\left(\varepsilon^{p}, T, \sigma_{M}, p\right), \\
& \varphi\left(\varepsilon^{p}, T, \sigma_{M}, p\right)=\varphi_{s}\left(\varepsilon^{p}, T, \sigma_{M}\right)+\left\{\varphi_{m}\left(\varepsilon^{p}, T\right)-\varphi_{s}\left(\varepsilon^{p}, T, \sigma_{M}\right)\right\} \exp \left(-\frac{<p-p_{R}>}{Q}\right)
\end{aligned}
$$

where $\sigma$ is the stress,$\sigma_{Y}$ is the yield stress, $\varepsilon^{p}$ is the plastic strain during the equivalent monotonic loading, $T$ is the temperature, $\sigma_{M}$ is the memory stress which is defined as the maximum equivalent stress that ever existed in the past with a view to prescribing cyclic properties, $p$ is the accumulated plastic strain, and $\varphi$ is the over stress (defined here the amount of stress above the yield stress) function which varies from monotonic property $\varphi_{m}$ to steady state cyclic property $\varphi_{s}$ asymptotically with the increase of $p$. Here, $\varphi$ is primarily a function of $\varepsilon^{p}$ and $p$, but includes $T, \sigma_{M}$ as parameters, $p_{R}$ is the accumulated plastic strain during the initial monotonic loading, $Q$ is a material constant governing the rate of cyclic hardening, and $<>$ indicates the MaCauley bracket.

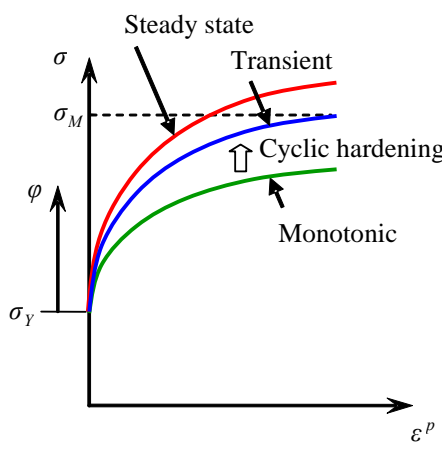

Fig.3: Generic stress-plastic strain relation
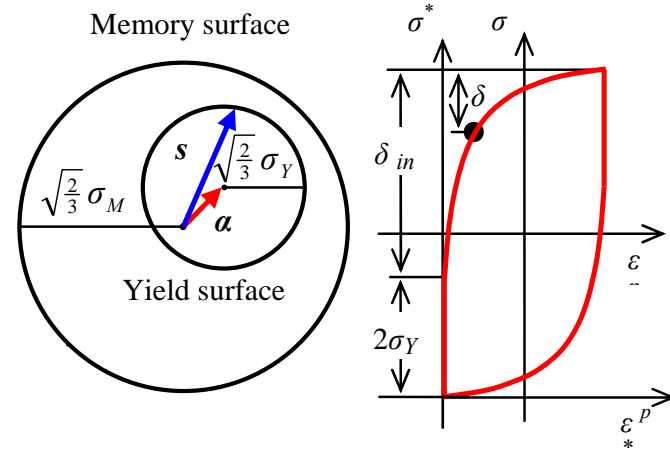

Fig. 4: Two-surface model

The generic stress-plastic strain relation under a certain state of temperature, memory stress, and accumulated plastic strain can produce hardening properties during the process from monotonic to cyclic steady state loading. The features of this modeling is lies in that it can express cyclic hardening with only one material constant, $Q$, and can generate the steady state cyclic behavior completely consistent with the fundamental material properties. 


\section{Two-surface cyclic plasticity model (TCP model)}

The concept of the two-surface model is shown in Fig. 4. The earlier model [3] is developed using a yield surface which moves kinematically in the deviatoric stress space, a stress memory surface, or a bounding surface, which grows in an isotropic fashion whenever the yield surface comes into contact with it, and a plastic hardening modulus function which is defined consistently with fundamental stress-strain characteristic equations. In this project, this model was extended to allow for arbitrary nonlinear stress-strain curves as well as temperature dependence of material properties by incorporating the modeling of cyclic hardening described above [4,5]. The developed model is called TCP model.

The yield surface using the von Mises yield function and the memory surface are expressed by

$$
f=\frac{3}{2}(\boldsymbol{s}-\boldsymbol{\alpha}):(\boldsymbol{s}-\boldsymbol{\alpha})-\sigma_{Y}^{2}=0, \quad F=\frac{3}{2} \boldsymbol{s}^{*}: \boldsymbol{s}^{*}-\sigma_{M}^{2}=0
$$

where $s, \alpha$ and $s^{*}$ are, respectively, the deviatoric stress tensor, the back stress tensor specifying the yield surface center and the deviatoric stress tensor on the memory surface, and $\sigma_{Y}$ and $\sigma_{M}$ represent the yield stress and the memory stress respectively. The yield surface moves inside (including contact) the memory surface, changing its size depending on temperature, while the memory surface grows isotropically only when contacted by the yield surface, according to the hardening properties depending on its own size (memory stress) and accumulated plastic strain. The memory stress under isothermal conditions is defined as the maximum equivalent stress in the previous loading history, but it should be updated step by step under varying temperature as

$$
\sigma_{M}=\operatorname{Max}\left\{\sigma_{M T}, \sigma_{e q}\right\}
$$

where $\sigma_{M T}$ is the memory stress varied due to temperature change (increment) and is evaluated as the stress value on the generic stress-strain curve after temperature change at the strain position (memory strain) corresponding to the memory stress before temperature change, and $\sigma_{e q}$ is the equivalent stress after increment.

Considering the effects by temperature change and cyclic hardening, the translation rule is assumed as

$$
\dot{\boldsymbol{\alpha}}=\mu \boldsymbol{\tau}+\frac{1}{\varphi}\left(\frac{\partial \varphi}{\partial T} \dot{T}+\frac{\partial \varphi}{\partial p} \dot{p}\right) \boldsymbol{\alpha}
$$

where $\tau$ is the translation direction vector during plastic loading, $\mu$ is the coefficient specifying its magnitude which is determined by the consistency condition during plastic loading. In the evaluation of tangent modulus, plastic strain rate tensor $\dot{\boldsymbol{\varepsilon}}^{p}$ is taken for $\tau$, while, in the back stress evaluation after increment calculation, the translation direction vector is taken in such a way that the yield surface and the memory surface come into contact tangentially at the point of intersection of the direction of $\dot{s}$ with the memory surface.

The constitutive equation of the model can be expressed in the rate form as

$$
\dot{\boldsymbol{\sigma}}=\boldsymbol{D}^{e p}:\left(\dot{\boldsymbol{\varepsilon}}-\dot{\boldsymbol{\varepsilon}}^{\theta}\right)+\dot{\boldsymbol{\sigma}}_{a}
$$

where $\dot{\boldsymbol{\sigma}}$ is the stress rate tensor, $\dot{\boldsymbol{\varepsilon}}$ is the strain rate tensor, $\dot{\boldsymbol{\varepsilon}}^{\theta}$ is the thermal strain rate tensor, $\dot{\boldsymbol{\sigma}}_{a}$ is the apparent stress rate tensor derived from temperature dependency of material properties, the notation “:” represents the scalar product of two tensors, and $\boldsymbol{D}^{e p}$ the elasto-plastic modulus tensor expressed as

$$
\boldsymbol{D}^{e p}=\boldsymbol{D}^{e}-\frac{18 G^{2}}{S_{0} \sigma_{Y}}(\boldsymbol{s}-\boldsymbol{\alpha}) \otimes(\boldsymbol{s}-\boldsymbol{\alpha}),
$$

with

$$
S_{0}=2\left(3 G+H_{k}+H_{c}\right) \sigma_{Y} .
$$

where $\boldsymbol{D}^{e}$ is the elastic modulus tensor, $G$ is the elastic shear modulus, $H_{k}\left(=\partial \varphi / \partial \varepsilon^{p}\right)$ is the kinematic hardening modulus at the current stress position measured by the distance $\delta$ in Fig. $4, H_{c}$ is the additional kinematic hardening modulus due to cyclic hardening (or softening) derived from $\partial \varphi / \partial p$, and the notation $\otimes$ denotes the tensor product.

\section{Multi-linear cyclic plasticity model (MCP model)}

The multi-linear cyclic plasticity model (MCP model) was developed as an extension of the MK model in much the same way as the two-surface cyclic plasticity model mentioned above [6]. The back stress tensor is assumed to be a sum of $\mathrm{M}$ back stress components. The kinematic hardening rule for the $\mathrm{m}$-th component proposed by Ohno and Wang [2] was extended in consideration of cyclic hardening as well as temperature dependency of plastic modulus as 


$$
\dot{\alpha}_{(m)}=\frac{2}{3} H_{(m)} \dot{\varepsilon}^{p}+\frac{1}{H_{(m)}}\left(\frac{\partial H_{(m)}}{\partial T} \dot{T}+\frac{\partial H_{(m)}}{\partial p} \dot{p}\right) \alpha_{(m)}-U\left(g_{(m)}\right) \frac{H_{(m)}}{r_{(m)}^{2}}<\dot{\varepsilon}^{p}: \alpha_{(m)}>\alpha_{(m)}
$$

where $H_{(m)}$ and $r_{(m)}$ are respectively the kinematic hardening coefficient and the threshold limit for back stress element $(m), g_{(m)}$ is the function expressed by $g_{(m)}=\frac{3}{2}\left(\boldsymbol{\alpha}_{(m)}: \boldsymbol{\alpha}_{(m)}\right)-r_{(m)}^{2}$, and $U(\mathrm{)}$ indicate the unit step function. In Eq.(10), the first term describes linear kinematic hardening, the second term represents hardening due to temperature change and increase of accumulated plastic strain, and the third term comes from the condition that the back stress component would not exceed its threshold limit.

The memory stress is adopted as a parameter to specify the generic stress-plastic strain relation in the same manner as in the above two-surface cyclic plasticity model. Using the yield surface of Eq.(3), the resulting constitutive equation of MCP model becomes exactly the same as that of TCP model, i.e., Eqs.(8) and (9). It is noted, however, that the kinematic hardening moduli in the MCP model are expressed by a sum of components as

$$
H_{k}=\sum_{m=1}^{M} H_{(m)}\left\{1-U\left(g_{(m)}\right)\left(\frac{3}{2 \sigma_{Y} r_{(m)}}\right)^{2}<B_{(m)}>B_{(m)}\right\}, \quad H_{c}=\sum_{m=1}^{M}\left(\frac{3 B_{(m)}}{2 H_{(m)} \sigma_{Y}}\right) \frac{\partial H_{(m)}}{\partial p}
$$

with

$$
B_{(m)}=(s-\alpha): \boldsymbol{\alpha}_{(m)}
$$

\section{APPLICATION ANALYSES BY USING DEVELOPED CONSTITUTIVE MODELS}

\section{Analyses of Experiments}

Experiments consist of three categories, i.e., uni-axial tests, basic structure element tests, and structure model tests [1]. The uni-axial tests consist of two series of tests, i.e., material property test and temperature dependency test. The former is used to mainly determine the parameters of constitutive models, and latter is used for basic verification of temperature dependency performance. The basic structure element tests consist of two kinds of simple ratcheting tests, i.e., three bar ratcheting test and bi-axial ratcheting test. The former is based on the famous two bar ratcheting theory. The latter was proposed considering the results of the preliminary analysis. In the structure model tests, similar phenomenon as the ratcheting in the reactor vessel is realized. Small cylinders are used and the movement of temperature distribution is generated by heater and water level control. These experiments are recategorized into two groups, i.e., verification test problems and design applicability test problems for the assessment of constitutive models as shown in Table 1[7]. In this paper some results of these problems are described.

Table 1: Verification and validation problems for constitutive models

\begin{tabular}{|c|l|}
\hline No. & \multicolumn{1}{|c|}{ Title } \\
\hline Verification test problems \\
\hline T-1-1 & Stress-strain relation curve description test \\
\hline T-2-1 & Cyclic behavior description test (without cyclic hardening) \\
\hline T-2-2 & Cyclic behavior description test (with cyclic hardening) \\
\hline T-3-1 & Simple tension test with temperature change \\
\hline T-3-2 & Cyclic axial strain-temperature in-phase test (without cyclic hardening) \\
\hline T-3-3 & Cyclic axial strain-temperature out of-phase test (without cyclic hardening) \\
\hline T-3-4 & Cyclic axial strain-temperature in-phase test (with cyclic hardening) \\
\hline T-3-5 & Cyclic axial strain-temperature out of-phase test (with cyclic hardening) \\
\hline T-4-1 & Cyclic axial strain-constant pressure test \\
\hline T-4-2 & Cyclic axial strain-pressure in-phase test \\
\hline T-4-3 & Cyclic axial strain-pressure out of-phase test \\
\hline Design applicability test problems \\
\hline T-5-1 & Three bar thermal ratcheting test \\
\hline T-5-2 & Ratcheting test of cylinder with moving temperature distribution \\
\hline
\end{tabular}

Cyclic axial strain-temperature in-phase test (T-3-4)

Fig. 5(a) shows the diagrams of the temperature and the strain hysteresis of cyclic axial strain-temperature in-phase test. Fig. 5(b) shows the stress/strain hysteresis loop obtained by MCP model compared with the experimental result at 50th cycle. The cyclic hardening behavior is mainly observed in high temperature side (tension side) from the analysis. Some discrepancy can be observed between the analysis result and the experimental 
one, which might be mostly due to the representativeness of the cyclic properties used for the tested material, especially around 200C, but the analysis gives conservative result from the point of evaluation of strain range [6].

Cyclic axial strain-pressure in-phase test (T-4-2)

Fig. 6(a) shows the concept of the test. The axial strain and circumferential stress are induced by the actuator and internal pressure controlled independently. Fig. 6(b) shows the circumferential strain increase corresponding to loading cycles in case of internal pressure-axial strain in-phase cyclic test. In the figure Gage 1 to 4 denote the results of experiment, and MCP and MK indicate the analysis results by MCP model and MK model, respectively [6]. Both analyses give enough conservative results, but that by MCP model is a little closer to the experimental result and the tendency of saturation is observed, while it cannot in the case of MK model.

\section{Three bar thermal ratcheting test (T-5-1)}

Fig. 7(a) shows the test specimen of the three bar thermal ratcheting test. The center bar is subjected to cyclic thermal loading by heater and water jacket, and primary load can be added by actuator. Fig. 7(b) shows the relation between the accumulated axial strain and number of cycles obtained by one of the tests with the analysis results by several plasticity models. MK-SRR model again gives very conservative result and TCP model coincides very well with experimental result, while other two models cannot describe the ratcheting behavior [7].

\section{Ratcheting test of cylinder with moving temperature distribution (T-5-2)}

Fig. 8(a) shows the test apparatus. The coil heater and the water surface level are fixed. The movement of temperature distribution subjected to the cylindrical specimen can be realized by moving the specimen up and down. Fig. 8(b) shows the relation between the accumulated axial strain and number of cycles obtained by the test with the analysis results by three plasticity models. The solid lines denote the models determined by the minimum trend curves of stress-strain relations from the database of 316FR and the dashed lines denote the ones determined by the average trend curves. As can be seen from the figure, all models give conservative predictions, but MK-SRR model gives too much conservative result. MK model gives closer prediction to the experimental result than TCP model and the strains predicted by TCP model are about twice of those by MK model [7].

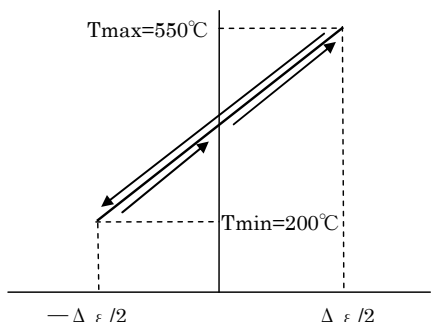

(a) Temperature and strain hysteresis

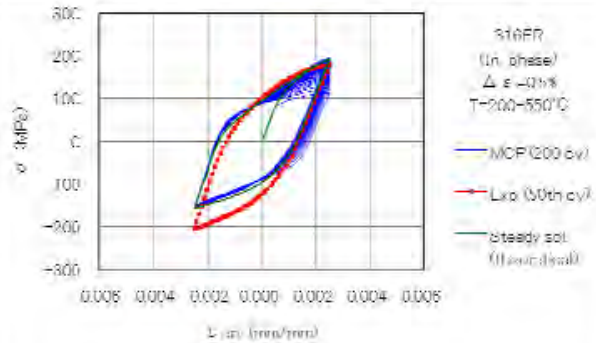

(b) Simulation results

Fig. 5: Cyclic tests with temperature and strain hysteresis

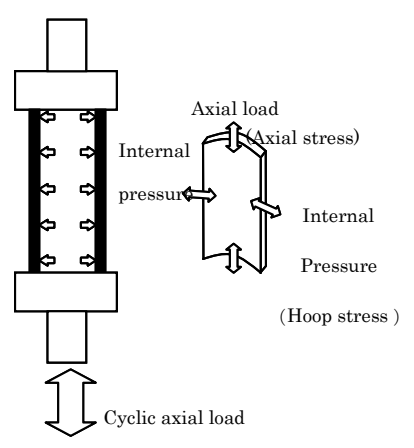

(a) Test apparatus

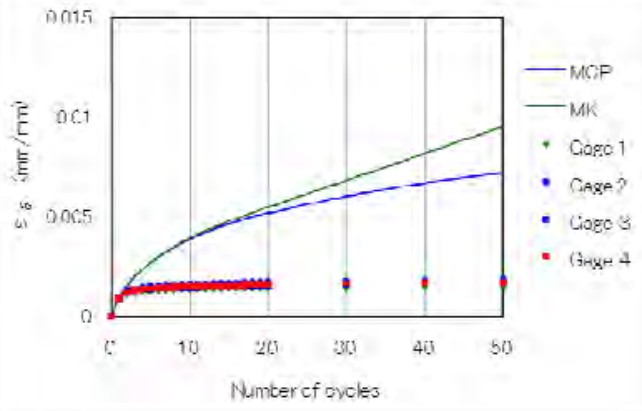

(b) Circumferential strain vs loading cycle

Fig. 6: Axial strain-pressure in-phase cyclic test 


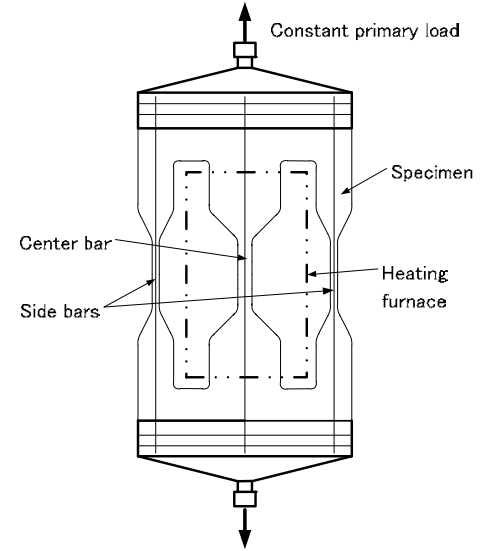

(a) Test specimen

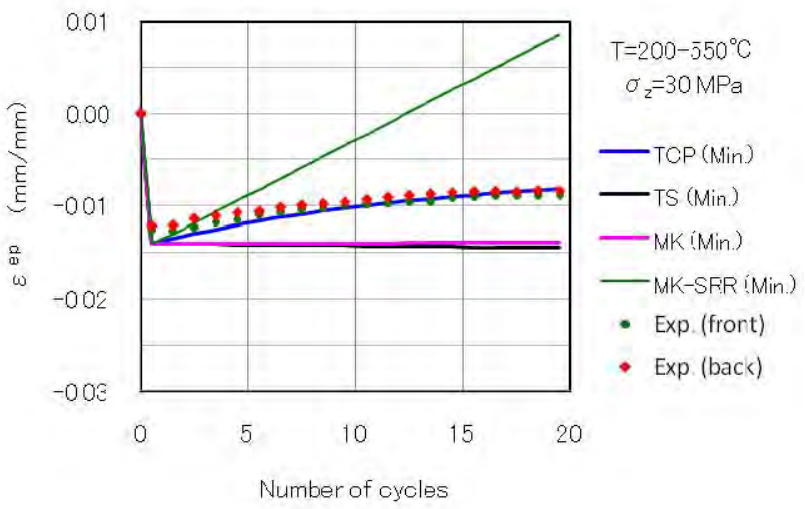

(D) Axıaı strain vs number or cycies

Fig. 7: Three bar thermal ratcheting test

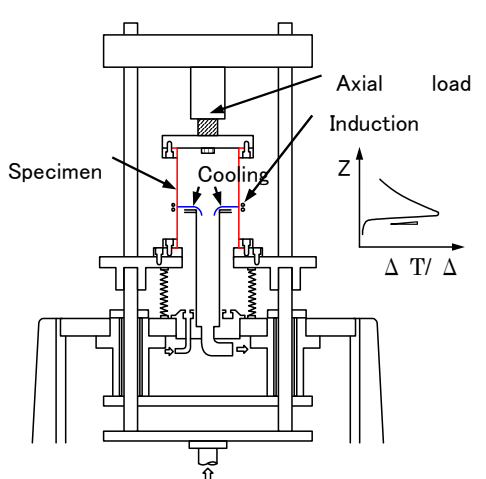

(a) Test apparatus

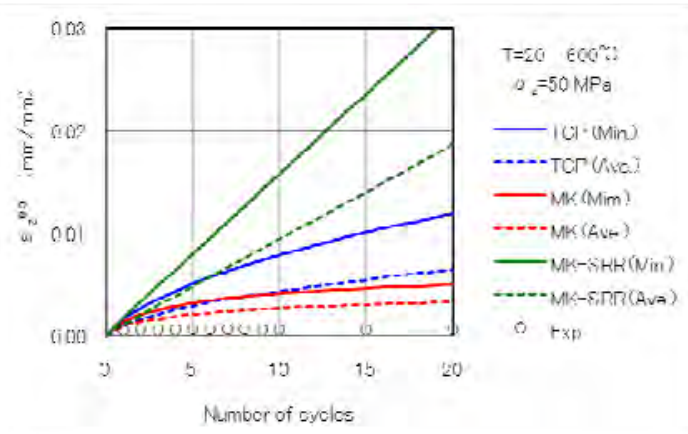

(b) Axial strain vs loading cycle

Fig. 8: Ratcheting test of cylinder with moving temperature distribution

\section{Analyses of Reactor Vessel Model}

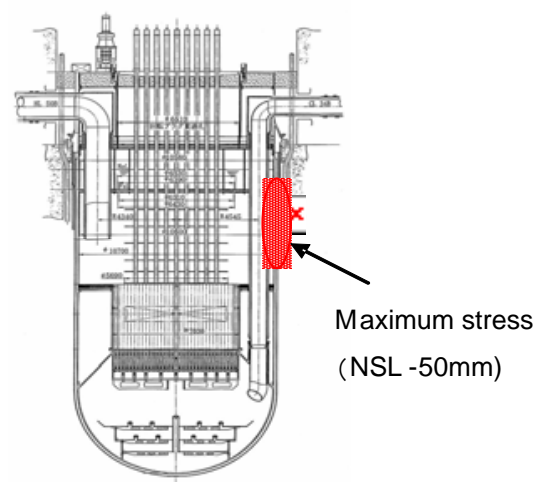

Demonstration FBR plant design : $500 \sim 750 \mathrm{MWe}$ (tentative)

Thickness : $\quad 30 \mathrm{~mm} \sim 60 \mathrm{~mm}$

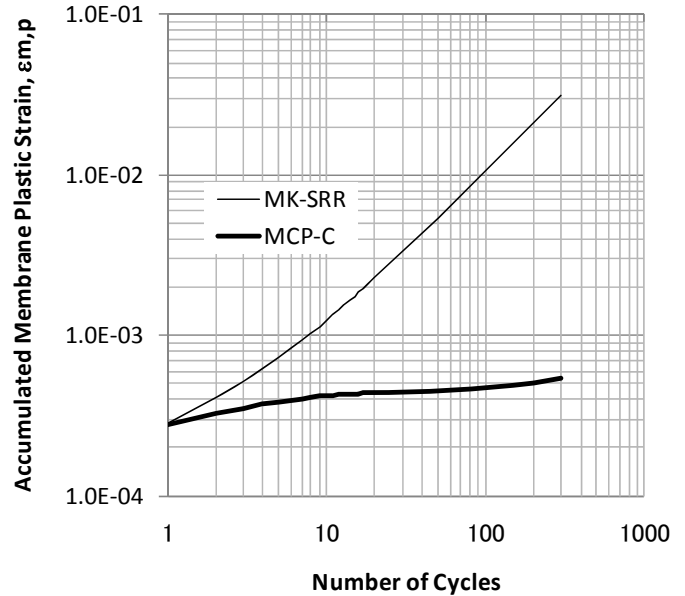

(b) Accumulated membrane plastic strain vs cycles

Fig. 9: Analyses of reactor vessel model 
Firstly, thermal conduction analysis was carried out. Then, structural analyses were carried out by using temperature distribution and its history obtained by the thermal conduction analysis [8]. The straight portion of the reactor vessel from NsL-3000mm to NsL+1600mm was modeled by axisymmetric elements(Fig. 9(a)). Inner radius is $5350 \mathrm{~mm}$. Material is $316 \mathrm{FR}$. The case with $60 \mathrm{~mm}$ in thickness, internal pressure and weight was picked up. Fig. 9(b) shows the accumulated maximum membrane strain with thermal cycles. The analysis result by MK-SRR model is also plotted. The accumulated strain at 300 cycles by MK-SRR model is about 3\%. While that by MCP model stay around $0.05 \%$. This means that the ratcheting strain is restrained with cycles by the effect of cyclic hardening in the case of cyclic hardening materials like 316FR.

\section{CONCLUSION}

In order to develop rational constitutive models that enable prediction of complex inelastic behaviors of reactor vessel and to prepare the design guide based on inelastic analysis, this R\&D were implemented from 2006FY to 2009FY. In this paper, the details of developed high-accuracy plasticity models, its verification by tests and the application to the evaluation of accumulated strain of design reactor vessel were mainly introduced following the previous paper [1]. It was found that the high-accuracy plasticity models well predicted the results of experiments and the simplified plasticity model without cyclic hardening was convenient but too conservative.

Based on the R\&D results, the draft of "Design Guide based on Inelastic Analyses" was prepared [9]. It describes the requirements for constitutive models and the criteria to check whether the requirements are satisfied or not, providing a set of verification and design applicability test problems for constitutive models and the details of the developed models.

\section{Acknowledgements}

Present study includes the result of "Development of elevated temperature structural design method for fast reactor vessels and failed fuel detection and location system" entrusted to JAEA by the Ministry of Education, Culture, Sports, Science and Technology of Japan (MEXT). The authors are grateful to Mr. M. Ando, Dr. S. Okajima of JAEA, Mr. K. Kikuchi, Dr. M. Yamauchi of MHI, and Mr. T. Chiba, Y. Karakida, I.Furuhashi of CTC.

\section{REFERENCES}

[1] Tsukimori, K., Iwata, K., Kawasaki, N., Yada, H., and Kasahara, N., "Development of constitutive models for fast reactor design -Strategy of the study and results in the first half stage-”, International Conference on Structural Mechanics in Reactor Technology, Espoo, Finland, SMiRT20 -1677, August, 2009.

[2] Ohno, N., Wang, J.D., "Kinematic hardening rule with critical state of dynamic recovery, Part I", International Journal of Plasticity. Vol. 9, 1993, pp. 375-390.

[3] Iwata, K., "A two-surface cyclic plasticity model consistent with fundamental stress-strain equations of the power-law type”, Journal of Nuclear Engineering and Design, Vol. 139, 1993, pp. 319-326.

[4] Iwata, K., Tsukimori, K., Kasahara, N. and Kawasaki, N., "Development of evaluation method based on inelastic analyses for structural design of fast reactor components (2) Summary of recommended constitutive models”, M\&M 2010 conference of JSME, Nagaoka, Japan, GS-018 October, 2010. (in Japanese)

[5] Iwata, K., “A temperature dependent two-surface cyclic plasticity model”, M\&M 2009 conference of $J S M E$, Sapporo, Japan, OS0202, July, 2009. (in Japanese)

[6] Iwata, K., “A temperature dependent multi-linear cyclic plasticity model”, M\&M 2011 conference of JSME, Kitakyushu, Japan, OS1202, July, 2011.(in Japanese)

[7] Iwata, K., Tsukimori, K., and Kawasaki, N., "Development of evaluation method based on inelastic analyses for structural design of fast reactor components (3)Verification and validation of constitutive models”, M\&M 2010 conference of JSME, Nagaoka, Japan, GS-019, October, 2010.(in Japanese)

[8] Tsukimori, K., Okajima, S.. Ando, M., Kasahara, N. and Kawasaki, N., "Development of evaluation method based on inelastic analyses for structural design of fast reactor components (4) Examples of design analyses by use of the guideline”, M\&M 2010 conference of JSME, Nagaoka, Japan, GS-020 October, 2010. (in Japanese)

[9] Tsukimori, K., Iwata, K., Kawasaki, N. and Kasahara, N., "Development of evaluation method based on inelastic analyses for structural design of fast reactor components (1) Guideline of inelastic design analysis”, M\&M 2010 conference of JSME, Nagaoka, Japan, GS-017 October, 2010. (in Japanese) 